Nephrologe 2021 · 16:360-371

https://doi.org/10.1007/s11560-021-00527-w

Angenommen: 23. Juli 2021

Online publiziert: 30. August 2021

๑) Springer Medizin Verlag GmbH, ein Teil von

Springer Nature 2021

\section{Redaktion}

Uwe Heemann, München

Hans-Joachim Anders, München

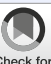

\title{
Strategien zur effektiven und nebenwirkungsarmen Therapie ANCA-assoziierter Vaskulitiden
}

\author{
Ulf Schönermarck ${ }^{1}$. Volker Vielhauer ${ }^{2}$ \\ 'Medizinische Klinik und Poliklinik IV, Nephrologisches Zentrum, Campus Großhadern, LMU Klinikum, \\ München, Deutschland \\ ${ }^{2}$ Medizinische Klinik und Poliklinik IV, Nephrologisches Zentrum, Campus Innenstadt, LMU Klinikum, \\ München, Deutschland
}

\section{Zusammenfassung}

Die Pathogenese von ANCA(antineutrophile zytoplasmatische Antikörper)-assoziierten Vaskulitiden (AAV) ist komplex, das bessere Verständnis der letzten Jahre hat jedoch neue therapeutische Ansätze ermöglicht. In den letzten Jahren stand die Minimierung toxischer Therapieeffekte im Vordergrund. In der Remissionsinduktion stehen für die schwere Granulomatose mit Polyangiitis (GPA) und die mikroskopische Polyangiitis (MPA) neben Glukokortikoiden Cyclophosphamid und Rituximab zur Verfügung. Die aktuellen Empfehlungen ermöglichen eine raschere Reduktion der Steroiddosis und sehen den Einsatz der Plasmapherese zurückhaltend. Zur Remissionserhaltung stehen Rituximab und Azathioprin zur Verfügung. Medikamentenwahl und Dauer der Remissionserhaltung richten sich insbesondere nach dem Rezidivrisiko. Der Stellenwert von niedrig dosierten Steroiden ist nicht endgültig geklärt. Neue Therapieansätze wie der C5a-Rezeptor-Inhibitor Avacopan könnten in Zukunft eine steroidminimierte Therapie ermöglichen. Die Therapie der eosinophilen Granulomatose mit Polyangiitis (EGPA) ist weniger evidenzbasiert und beinhaltet Glukokortikoide, Immunsuppressiva je nach Erkrankungsschwere und zunehmend Biologika (z. B. Interleukin[IL]-5-Blockade). Supportive Maßnahmen (Impfungen, Infektionsprophylaxe, kardiovaskuläres Risikomanagement) gewinnen an Bedeutung. Zukünftige Therapiestrategien müssen das individuelle Risiko (z. B. ANCA-Subtyp, Rezidivrisiko) für Auswahl und Dauer der Therapien besser berücksichtigen.

\section{Schlüsselwörter}

Antineutrophile zytoplasmatische Antikörper · Glukokortikoide · Granulomatose mit Polyangiitis · Mikroskopische Polyangiitis - Eosinophile Granulomatose mit Polyangiitis

\section{Einleitung}

Die mit dem Auftreten von ANCA(antineutrophile zytoplasmatische Antikörper)assoziierten Kleingefäßvaskulitiden (AAV) sind eine Gruppe von potenziell lebensbedrohlichen Autoimmunerkrankungen, die durch die Entwicklung von Autoantikörpern gegen die Proteasen Proteinase 3 (PR3-ANCA) oder Myeloperoxidase (MPO-ANCA) von neutrophilen Granulozyten charakterisiert sind. Klinische, histologische und pathophysiologische Unterschiede erlauben eine Unterteilung in Granulomatose mit Polyangiitis (GPA), mikroskopische Polyangiitis (MPA) und eosinophile Granulomatose mit Polyangiitis (EGPA; [18]; - Tab. 1).

Pathophysiologisch liegt den AAV eine endotheliale Schädigung kleiner Gefäße zugrunde, die durch überschießende Aktivierung zirkulierender neutrophiler Granulozyten nach Bindung von ANCA verursacht wird. Dies führt zu einer nekrotisierenden Vaskulitis mit entzündlicher und ischämischer Gewebeschädigung, fibrotischem Gewebeumbau und zunehmendem Funktionsverlust betroffener Organe. Daneben sind GPA und EGPA durch eine neu- 
Tab. 1 Gegenüberstellung der ANCA(antineutrophile zytoplasmatische Antikörper)-assoziierten Vaskulitiden. (Mod. nach [22])

\begin{tabular}{|l|l|l|l|l|l|}
\hline & $\begin{array}{l}\text { Prävalenz } \\
\text { (pro 1 Mio. } \\
\text { Einwohner) }\end{array}$ & ANCA-Positivität & Organmanifestationen & $\begin{array}{l}\text { Nieren- } \\
\text { beteiligung } \\
\text { (in \%) }\end{array}$ & $\begin{array}{l}\text { RPGN } \\
\text { (in } \\
\%)\end{array}$ \\
\hline GPA & $59-98$ & $\begin{array}{l}\sim 90 \% \\
(75 \% \text { PR3-ANCA, } \\
20 \% \text { MPO-ANCA })\end{array}$ & $\begin{array}{l}\text { HNO, Lunge, Nieren, } \\
\text { Gelenke, Augen } \\
\text { HNO-limitierte Verlaufs- } \\
\text { form }\end{array}$ & $\sim 70$ & $\sim 50$ \\
\hline EGPA & $7-24$ & $\begin{array}{l}\sim 40 \% \\
(<5 \% \text { PR3-ANCA, } \\
30-40 \% \text { MPO- } \\
\text { ANCA) }\end{array}$ & $\begin{array}{l}\text { Lunge (Asthma), HNO, } \\
\text { PNP, Herz, Haut }\end{array}$ & $\sim 25$ & $<15$ \\
\hline MPA & $9-28$ & $\begin{array}{l}\sim 9 \% \% \\
(25 \% \text { PR3-ANCA, } \\
60 \% \text { MPO-ANCA })\end{array}$ & $\begin{array}{l}\text { Niere, Lunge } \\
\text { Renal-limitierte und } \\
\text { pulmonal-limitierte } \\
\text { Verlaufsformen }\end{array}$ & $>90$ & $\sim 65$ \\
& & & & \\
\hline
\end{tabular}

GPA Granulomatose mit Polyangiitis, EGPA eosinophile Granulomatose mit Polyangiitis, MPA mikroskopische Polyangiitis, HNO Hals/Nase/Ohren, PR3 Proteinase 3, MPO Myeloperoxidase, PNP Polyneuropathie, RPGN rapid-progressive Glomerulonephritis

trophilen- bzw. eosinophilenreiche granulomatöse Entzündung charakterisiert.

Die AAV können sich im Bereich der kleinen Gefäße eines jeden Organs manifestieren, wobei GPA und MPA am häufigsten den oberen und unteren Respirationstrakt und die Nieren betreffen. Verläufe mit organlimitierter Manifestation können auftreten, häufig liegen jedoch schwere, die Organfunktion bedrohende oder vital bedrohliche Manifestationen vor. Neben Gemeinsamkeiten zeigen sich Unterschiede in Bezug auf klinische Manifestationen, ANCA-Spezifität, Pathogenese und Therapieansprechen. Allen AAV-Formen gemeinsam ist ihre Neigung zu Rezidiven, wobei das Rezidivrisiko bei der GPA am höchsten ist [22].

\section{Epidemiologie}

Mit einer Inzidenz von 12 Fällen/1 Mio. Einwohner/Jahr zählen AAV zu den seltenen Erkrankungen. Ihre Prävalenz wird in Deutschland mit etwa 150 je 1 Mio. Einwohner geschätzt ([12]; - Tab. 1). Epidemiologische Studien weisen auf deutliche geographische und ethnische Unterschiede hin. So treten GPA bzw. PR3ANCA+-AAV vorzugsweise in Populationen mit europäischen Wurzeln auf, während sie in Asien selten sind. Auch sinkt ihre Inzidenz in Richtung Äquatornähe. Dagegen dominiert in Asien die MPA bzw. MPO-ANCA+-AAV $[22,43]$. Dies spricht ne- ben Umwelteinflüssen für prädisponierende genetische Faktoren.

\section{Pathophysiologie}

Der klinischen Manifestation der AAV geht der Verlust der immunologischen Toleranz von autoreaktiven T- und B-Zellen gegenüber den Neutrophilenproteinen PR3 oder MPO voraus. Genomweite Assoziationsstudien (GWAS) weisen darauf hin, dass genetische Polymorphismen spezifisch zum Toleranzverlust prädisponieren [22] und die induzierte Autoimmunität gegen PR3 oder MPO mit ihrer charakteristischen Autoantikörperbildung unter dem Einfluss weiterer Umweltfaktoren und immunologischer Effektormechanismen die phänotypisch überlappenden klinischen Krankheitsbilder der GPA oder MPA auslöst. ANCA binden an zirkulierende Neutrophile und aktivieren diese. Autoreaktive Tund B-Zellen können auch direkt zum inflammatorischen Gewebeschaden beitragen. ANCA-aktivierte Neutrophile produzieren vermehrt den B-Zell-aktivierenden Faktor BAFF („B-lymphocyte stimulator", BLyS), sodass autoreaktive B-Zellen und Plasmablasten anhaltend aktiviert werden [22]. Im Gegensatz zu B-Zell-depletierenden Therapien sind Therapieansätze zur Beeinflussung des Toleranzverlustes bisher nicht verfügbar.

ANCA-aktivierte Neutrophile führen zur endothelialen Schädigung in vulnerablen Gefäßregionen, die sich als nekrotisieren- de Kleingefäßvaskulitis mit lokaler Inflammation, Gewebedestruktion und Organischämie manifestiert. Glomeruläre und pulmonale Kapillaren sind besonders betroffen, die zugrunde liegenden Mechanismen dieser Präferenz sind nicht verstanden. Ebenso ist unklar, warum Granulome bei PR3-ANCA-assoziierter AAV, aber praktisch nicht bei MPO-ANCA+-AAV auftreten.

Die ANCA-Bindung an Neutrophile induziert durch vermehrte Expression von Adhäsionsmolekülen, Veränderungen des Zytoskeletts und Bildung reaktiver Sauerstoffradikale inflammatorische Effektorfunktionen. Inflammatorische Mediatoren werden durch Degranulation, Bildung neutrophiler extrazellulärer Netze („neutrophil extracellular traps“, NET) und Abgabe von Mikropartikeln freigesetzt und führen zur Gefäßschädigung [22]. Dies aktiviert den alternativen Weg des Komplementsystems mit Bildung des Komplementspaltprodukts C5a. C5a führt über Bindung an den auf ihnen exprimierten C5a-Rezeptor zur Aktivierung von Neutrophilen, fördert ihre Retention im entzündeten Kapillarbett und hält damit das lokale Entzündungsgeschehen selbstverstärkend aufrecht [45]. C5a verstärkt auch die T-Zell-Antwort durch Aktivierung dendritischer Zellen. Die zentrale Bedeutung von $\mathrm{C} 5 \mathrm{a}$ wird durch die erfolgreiche Etablierung einer C5a-Rezeptorblockierenden Therapie unterstrichen.

Die Pathogenese der EGPA ist weniger gut verstanden. Unterschiede in Genetik, klinischem Verlauf und Therapieansprechen implizieren zumindest teilweise unterschiedliche pathophysiologische Mechanismen der ANCA+- und ANCA--EGPA [22]. Proliferation und Aktivierung eosinophiler Granulozyten werden durch Interleukin(IL)-5 und IL-13 vermittelt [22], entsprechend konnte die IL-5-Blockade therapeutische Erfolge bei der EGPA erzielen.

\section{Diagnostik}

Die Diagnose einer AAV wird in Zusammenschau von Klinik, Labor, Bildgebung, Autoantikörperdiagnostik und Histologie gestellt. Zentraler Bestandteil ist die ANCA-Testung. Aktuelle Konsensusempfehlungen sehen bei hoher klinischer Prätestwahrscheinlichkeit den primären Einsatz antigenspezifischer Immunoassays 
Tab. 2 Stadieneinteilung der ANCA(antineutrophile zytoplasmatische Antikörper)-assoziierten Vaskulitis nach Empfehlungen der European Vasculitis Society (EUVAS) und der European League Against Rheumatism (EULAR). (Nach [27])

\begin{tabular}{|c|c|c|c|c|}
\hline $\begin{array}{l}\text { Aktivitäts- } \\
\text { stadien }\end{array}$ & EUVAS/EULAR-Definition & $\begin{array}{l}\text { Systemische Vaskulitis } \\
\text { außerhalb HNO bzw. Lunge }\end{array}$ & $\begin{array}{l}\text { Vitale Organ- } \\
\text { funktion bedroht }\end{array}$ & Serumkreatinin \\
\hline Lokalisiert & $\begin{array}{l}\text { Oberer/unterer Respirationstrakt ohne systemische Manifes- } \\
\text { tation, ohne B-Symptomatik, ANCA häufig negativ }\end{array}$ & Nein & $\mathrm{Ja}$ & $\begin{array}{l}<120 \mu \mathrm{mol} / \mathrm{l} \\
(<1,4 \mathrm{mg} / \mathrm{dl})\end{array}$ \\
\hline $\begin{array}{l}\text { Früh-sys- } \\
\text { temisch }\end{array}$ & $\begin{array}{l}\text { Alle Organbeteiligungen möglich, nicht lebens- oder organ- } \\
\text { bedrohend }\end{array}$ & $\mathrm{Ja}$ & $\mathrm{Ja}$ & $\begin{array}{l}<120 \mu \mathrm{mol} / \mathrm{l} \\
(<1,4 \mathrm{mg} / \mathrm{dl})\end{array}$ \\
\hline Generalisiert & $\begin{array}{l}\text { Renale Beteiligung oder andere organbedrohende Manifes- } \\
\text { tation }\end{array}$ & $\mathrm{Ja}$ & $\mathrm{Ja}$ & $\begin{array}{l}<500 \mu \mathrm{mol} / \mathrm{l} \\
(<5,8 \mathrm{mg} / \mathrm{dl})\end{array}$ \\
\hline Schwer & Renale Beteiligung oder anderes Organversagen & $\mathrm{Ja}$ & Organversagen & $\begin{array}{l}>500 \mu \mathrm{mol} / \mathrm{l} \\
(>5,8 \mathrm{mg} / \mathrm{dl})\end{array}$ \\
\hline Refraktär & $\begin{array}{l}\text { Progressiver Krankheitsverlauf, refraktär auf Standardthera- } \\
\text { pie }\end{array}$ & $\mathrm{Ja}$ & $\mathrm{Ja}$ & Jedes \\
\hline
\end{tabular}

zum Nachweis von PR3-ANCA bzw. MPOANCA als Screeninguntersuchung vor [2]. Eine Nierenbiopsie stellt weiterhin den Goldstandard für die Diagnose bei Erstmanifestation, bei Rekurrenz und als prognostischer Marker dar, sollte den Therapiebeginn aber nicht verzögern.

\section{Beurteilung von Krankheits- aktivität und Organschädigung}

Für individualisierte Therapieentscheidungen sind eine Beurteilung von Krankheitsaktivität, präexistenten Organschäden, des Risikos für Rezidive und irreversible Organschäden sowie von Gesamtprognose und Lebensqualität erforderlich. Die EUVAS(European Vasculitis Society)-Einteilung in 5 Stadien anhand von Aktivität und Krankheitsschwere hat sich bewährt ([27]; - Tab. 2). Im klinischen Alltag wird jedoch häufig nur nach Vorliegen oder Fehlen eines schweren Organbefalls bzw. drohenden Organverlustes (z. B. Glomerulonephritis, diffuse alveoläre Hämorrhagie) unterschieden.

Validierte Instrumente zur Bestimmung der Krankheitsaktivität stellen der Birmingham Vasculitis Activity Score (BVAS) für alle AAV-Formen sowie der Five-Factor Score (FFS) dar [22]. Chronische krankheits- und therapiebedingte Organschäden können mit dem Vasculitis Damage Index (VDI) erfasst werden [22]. Der Glucocorticoid Toxicity Index (GTI) erfasst Nebenwirkungen der Glukokortikoid(GC)-Therapie [25].

Risikofaktoren für eine erhöhte Mortalität sind:

- höheres Lebensalter,

- Nierenfunktionseinschränkung,
- pulmonale Hämorrhagien,

- hohe initiale Krankheitsaktivität im BVAS,

- Rezidivhäufigkeit,

- Infektionen,

- Vorliegen chronischer Organschäden im VDI.

Histologische Klassifikationen der AAV korrelieren mit dem renalen Outcome: Je mehr Glomerulosklerose, desto schlechter, je mehr normale Glomerula, desto besser [1]. Auch der FFS korreliert mit der Prognose, da die erfassten Parameter Alter, Niereninsuffizienz, kardiale Beteiligung, gastrointestinale Manifestationen und Fehlen einer HNO(Hals/Nase/Ohren)Beteiligung mit der Mortalität korrelieren [22].

\section{Prognose}

Bessere Diagnosemöglichkeiten und effektive immunsuppressive Therapien haben die einstmals fatale Prognose dramatisch verbessert. Von einer $90 \%$ igen Mortalität der unbehandelten GPA in den ersten 2 Jahren stieg die 5-Jahres-Überlebensrate auf 70-80\% und erreicht in einigen Kohorten das Niveau der Normalbevölkerung [8, 32]. Allerdings besteht im ersten Jahr nach Diagnosestellung weiterhin eine deutlich erhöhte Frühmortalität (11\%), wobei therapieassoziierte Nebenwirkungen als Ursache für ein Versterben im ersten Jahr der Behandlung 3-mal wahrscheinlicher sind als die direkten Folgen der Vaskulitis [24].

Aufgrund des zunehmend chronifizierten Verlaufs steigt auch das kardiovaskuläre Risiko, das insbesondere durch die
GC-Therapie erhöht wird [33]. Auch die Inzidenz von Malignomen ist bei AAV erhöht und wird durch die Wahl der immunsuppressiven Therapie maßgeblich beeinflusst. So ist eine Cyclophosphamid(CYC)Therapie ein wesentlicher Risikofaktor für die Entwicklung von Urothelkarzinomen und Nichtmelanom-Hauttumoren.

\section{Therapieprinzipien}

Eine rasche Diagnostik und Therapieeinleitung sind unerlässlich zur Verhinderung langfristiger chronischer Organschäden. Die Therapie der AAV ist biphasisch. Ziel der initialen Phase intensiverer Immunsuppression (Induktionstherapie für 3 bis 6 Monate) ist eine rasche, effektive Kontrolle der Krankheitsaktivität. Ihr folgt eine Phase weniger starker Immunsuppression zum Erhalt der Remission und zur Verhinderung von Rezidiven.

In beiden Phasen stellt das Erreichen einer effektiven Immunsuppression bei gleichzeitiger Minimierung toxischer Therapieeffekte eine anhaltende Herausforderung dar. In den letzten Jahren haben prospektive randomisierte Studien internationaler Studiennetzwerke wie der EUVAS, des Vasculitis Clinical Research Consortium bzw. der französischen Vaskulitis-Studiengruppe den Weg für effektive und gleichzeitig nebenwirkungsärmere Therapien eröffnet. Diese haben Einzug in aktuelle Therapieempfehlungen nationaler und internationaler Fachgesellschaften gefunden (u.a. der EULAR/ERA-EDTA [European Renal Association - European Dialysis and Transplant Association], S1-Leitlinie der Deutschen 


\begin{tabular}{|c|c|c|c|c|c|}
\hline $\begin{array}{l}\text { Therapie } \\
\text { (Studien- } \\
\text { protokoll) }\end{array}$ & Krankheitsstadium & Dosis & Ergebnis & Zu beachten & Zulassung \\
\hline \multicolumn{6}{|c|}{ Organbedrohendes Krankheitsstadium mit/ohne Organversagen } \\
\hline $\begin{array}{l}\text { CYC-Puls + GC } \\
\text { (CYCLOPS) [7, } \\
10]\end{array}$ & $\begin{array}{l}\text { Generalisiert, mit/ } \\
\text { ohne Organversa- } \\
\text { gen }\end{array}$ & $\begin{array}{l}15 \mathrm{mg} / \mathrm{kg} \mathrm{KG} \text { i.v. (max. } \\
1200 \mathrm{mg}, \text { Wochen } 0,2 \\
4,7,10,13 ; \text { alternativ } \\
0,5 \mathrm{~g} / \mathrm{m}^{2} \text { ) }\end{array}$ & $\begin{array}{l}\text { CYC-Puls ist oralem CYC nicht unter- } \\
\text { legen, } ~ 50 \% \text { der kumulativen CYC- } \\
\text { Dosis, weniger NW; im Langzeitverlauf } \\
\text { weniger Rezidive unter oralem CYC }\end{array}$ & $\begin{array}{l}\text { Dosisreduktion nach } \\
\text { Nierenfunktion und } \\
\text { Alter, Adjustierung } \\
\text { nach Leukozytennadir }\end{array}$ & $\begin{array}{l}\text { Formal nur } \\
\text { für GPA }\end{array}$ \\
\hline $\begin{array}{l}\text { Reduzierter } \\
\text { CYC-Puls + GC } \\
\text { (CORTAGE) } \\
{[29]}\end{array}$ & Alter $>65$ Jahre & $\begin{array}{l}\text { I.v.-CYC 6-mal } 500 \mathrm{mg} \\
\text { alle } 2 \text { bis } 3 \text { Wochen }\end{array}$ & $\begin{array}{l}\text { Im Vergleich zur Standardtherapie } \\
\text { vergleichbare Remissionsrate, weniger } \\
\text { NW }\end{array}$ & - & - \\
\hline $\begin{array}{l}\text { RTX+ GC } \\
\text { (RAVE, } \\
\text { RITUXVAS, } \\
\text { RITAZAREM) } \\
{[19,37]}\end{array}$ & $\begin{array}{l}\text { Generalisiert, mit/ } \\
\text { ohne Organversa- } \\
\text { gen }\end{array}$ & $\begin{array}{l}375 \mathrm{mg} / \mathrm{m}^{2} \text { i.v., Tage } 0, \\
7,14,21\end{array}$ & $\begin{array}{l}\text { RTX non-inferior zu CYC, vergleichbare } \\
\text { NW-Rate, RTX besser bei Pat. mit PR3- } \\
\text { ANCA }\end{array}$ & $\begin{array}{l}\text { „Rheuma-Schema": } 1 \mathrm{~g} \text {, } \\
\text { Tage } 0 \text { und } 14 \text {, formal } \\
\text { „off-label" }\end{array}$ & $\begin{array}{l}\text { Für schwere } \\
\text { GPA und MPA }\end{array}$ \\
\hline \multicolumn{6}{|c|}{ Alternativen bei früh-systemischem bzw. generalisiertem Stadium ohne Organversagen } \\
\hline $\begin{array}{l}\text { MTX+ GC } \\
\text { (NORAM) [6] }\end{array}$ & $\begin{array}{l}\text { Lokalisiert, früh- } \\
\text { systemisch }\end{array}$ & $\begin{array}{l}0,3 \mathrm{mg} / \mathrm{kg} \mathrm{KG}, 1-\mathrm{mal} \\
\text { pro Woche }(15-25 \mathrm{mg})\end{array}$ & $\begin{array}{l}\text { MTX non-inferior zu CYC, im Langzeit- } \\
\text { verlauf MTX mit mehr Rezidiven }\end{array}$ & $\begin{array}{l}\text { Folsäure am Tag nach } \\
\text { MTX-Gabe, kontraindi- } \\
\text { ziert bei Niereninsuffi- } \\
\text { zienz }\end{array}$ & $\begin{array}{l}\text { Formal "off- } \\
\text { label" }\end{array}$ \\
\hline $\begin{array}{l}M M F+G C \\
\text { (MYCYC) [20] }\end{array}$ & $\begin{array}{l}\text { Früh-systemisch, } \\
\text { generalisiert, kein } \\
\text { Organversagen } \\
\text { (eGFR }>15 \mathrm{ml} / \mathrm{min})\end{array}$ & 2-mal 1 g p.o. & $\begin{array}{l}\text { MMF non-inferior zu CYC, höhere Re- } \\
\text { zidivrate im Verlauf, v. a. bei PR3-ANCA }\end{array}$ & - & "Off-label" \\
\hline \multicolumn{6}{|c|}{ Glukokortikoidtherapie } \\
\hline $\begin{array}{l}\text { Methylpredni- } \\
\text { solonbolus } \\
\text { i.v. }\end{array}$ & $\begin{array}{l}\text { Schwere, organbe- } \\
\text { drohende Manifes- } \\
\text { tation }\end{array}$ & $\begin{array}{l}7-15 \mathrm{mg} / \mathrm{kg} \text { i.v. }(250 \\
\text { bis max. } 1000 \mathrm{mg}) \text { für } \\
3 \text { Tage }\end{array}$ & - & $\begin{array}{l}\text { Keine randomisierten } \\
\text { Studien, Stellenwert } \\
\text { umstritten }\end{array}$ & - \\
\hline $\begin{array}{l}\text { GC p.o. } \\
\text { (PEXIVAS) } \\
{[42]}\end{array}$ & $\begin{array}{l}\text { Alle Stadien, in } \\
\text { Kombination }\end{array}$ & $\begin{array}{l}\text { Beginn mit } 1 \mathrm{mg} / \mathrm{kg} \\
\text { (max. } 50-75 \mathrm{mg} / \mathrm{Tag}) \\
\text { p.o. }\end{array}$ & $\begin{array}{l}\text { Raschere Dosisreduktion mit weniger } \\
\text { NW bei gleicher Effektiviät }\end{array}$ & $\begin{array}{l}\text { Dosisreduktion im } \\
\text { Verlauf, ein Absetzen } \\
\text { ist mit vermehrten } \\
\text { Rezidiven verbunden }\end{array}$ & - \\
\hline $\begin{array}{l}\text { Avacopan } \\
\text { (CLEAR, } \\
\text { ADVOCATE) } \\
{[16,17]}\end{array}$ & $\begin{array}{l}\text { ANCA+-GPA und } \\
\text { MPA unter Therapie } \\
\text { mit CYC (gefolgt von } \\
\text { AZA) oder RTX }\end{array}$ & 2-mal $30 \mathrm{mg} \mathrm{p.o./Tag}$ & $\begin{array}{l}\text { Avacopan non-inferior zu GC nach } \\
6 \text { Monaten, überlegene Remissionsrate } \\
\text { nach } 12 \text { Monaten, weniger GC-NW }\end{array}$ & $\begin{array}{l}\text { Bisher keine Langzeit- } \\
\text { erfahrung }>12 \text { Monate }\end{array}$ & $\begin{array}{l}\text { Bisher nicht } \\
\text { zugelassen } \\
\text { bzw. nicht } \\
\text { verfügbar }\end{array}$ \\
\hline
\end{tabular}

Gesellschaft für Rheumatologie [DGRh] bzW. KDIGO[Kidney Disease: Improving Global Outcomes]-Leitlinien 2021 zur Glomerulonephritis; [11, 46]; - Tab. 3 und 4; - Abb. 1).

Aufgrund von Unterschieden hinsichtlich Pathogenese, klinischer Manifestationen, ANCA-Positivität und Therapieoptionen zwischen GPA und MPA im Vergleich zur EGPA werden diese getrennt dargestellt.

\section{Remissionsinduktion bei GPA und MPA}

Die Auswahl der Therapien richtet sich nach dem Auftreten organ- oder lebensbe- drohlicher Manifestationen, nach der Unterscheidung zwischen Erstmanifestation und Rezidiv, nach Risikofaktoren für einen rezidivierenden Verlauf, nach dem ANCASubtyp (PR3- vs. MPO-ANCA) sowie individuellen Faktoren wie Alter und Begleiterkrankungen (• Tab. 3; • Abb. 1).

\section{Remissionsinduktion bei potenziell organbedrohenden oder lebensbedrohlichen Manifestationen}

Merke. Für organbedrohende oder lebensbedrohliche Manifestationen werden Cyclophosphamid oder der CD20-Antikörper
Rituximab in Kombination mit Glukokortikoiden eingesetzt.

\section{Remissionsinduktion mit Cyclophosphamid}

Die Remissionsinduktion mit CYC erfolgt über 3 bis 6 Monate. Die Ergebnisse randomisierter Studien der letzten Jahre haben zu einer deutlichen Reduktion der CYCDosis geführt, durch Umstellung von oraler Dauertherapie auf intravenöse Pulsgabe (CYCLOPS) und einen früheren Wechsel auf eine remissionserhaltende Therapie (CYCAZAREM). Bei vergleichbarer Effektivität konnte die kumulative CYC-Dosis um $50 \%$ reduziert werden $(<10 \mathrm{~g} ;[7,34])$. Dies 
Tab. 4 Cyclophosphamid(CYC)-Dosis-Reduktion bei i.v.-Pulstherapie nach Alter und Nierenfunktion. (Nach [27])

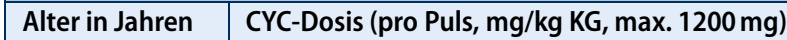

\begin{tabular}{|c|c|c|}
\hline & Kreatinin $<300 \mu \mathrm{mol} / \mathrm{l}(3,4 \mathrm{mg} / \mathrm{dl})$ & $\begin{array}{l}\text { Kreatinin } 300-500 \mu \mathrm{mol} / \mathrm{l} \\
(3,4-5,8 \mathrm{mg} / \mathrm{dl})\end{array}$ \\
\hline$<60$ & 15 & 12,5 \\
\hline $60-70$ & 12,5 & 10 \\
\hline$>70$ & 10 & 7,5 \\
\hline
\end{tabular}

ist im Hinblick auf dosisabhängige CYCNebenwirkungen (Knochenmarktoxizität, Fertilitätsrisiko, Tumorrisiko, Mortalität) ein deutlicher Vorteil, im Langzeitverlauf aber mit erhöhter Rezidivrate verknüpft [10]. Von den europäischen Leitlinien wird die i.v.-CYC-Pulstherapie favorisiert.

Merke. Eine geringere kumulative Cyclophosphamiddosis bedeutet weniger $\mathrm{Ne}$ benwirkungen, ist aber mit einem höheren Rezidivrisiko verbunden.

Für Patienten im gebär- bzw. zeugungsfähigen Alter sollte eine Kryokonservierung von Eizellen/Sperma bzw. für Frauen im gebärfähigen Alter eine Therapie mit $\mathrm{GnRH}$ (,ggonadotropin-releasing hormone")-Analoga angeboten werden.

\section{Cyclophosphamid-Dosisreduktion und Dialysetiming}

Bei eingeschränkter Nierenfunktion kumulieren CYC-Metabolite. Dies führt ebenso wie ein höheres Lebensalter zu vermehrten Komplikationen. Deshalb sollte eine Dosisreduktion nach Nierenfunktion und Alter erfolgen (• Tab. 4).

In einer randomisierten Studie (CORTAGE) konnte bei Patienten über 65 Jahre durch eine reduzierte CYC-Dosis (6-mal $500 \mathrm{mg}$ alle 2 bis 3 Wochen i.v.) und eine auf 9 Monate limitierte GC-Therapie eine Reduktion der Komplikationen bei gleicher Effektivität gezeigt werden [29], sodass diese Dosierung als Alternative für betagte Patienten in Frage kommt.

Bei Dialysepflichtigkeit muss eine Balance zwischen CYC-Toxizität durch Akkumulation und Unterdosierung durch effektive Elimination im Rahmen der Hämodialyse gehalten werden. Die Patienten sollten etwa $12 \mathrm{~h}$ nach der CYC-Gabe eine Dialysebehandlung erhalten, in der Regel am Tag nach der CYC-Gabe.

\section{Remissionsinduktion mit Rituximab}

Die Effektivität von Rituximab (RTX) wurde in randomisierten Studien gezeigt (RAVE, RITUXVAS; $[19,37])$ und führte 2013 zur Zulassung bei schwerer GPA und MPA. Im Vergleich zu CYC wird bei Patienten mit Rezidiv bzw. PR3-ANCA-Positivität bei gleicher Häufigkeit von Nebenwirkungen ein signifikant besseres Ansprechen erzielt [37, 40]. Nichtrandomisierte Studien und Subgruppenanalysen belegen eine vergleichbare Wirkung der alleinigen RTX-Gabe (ohne CYC) auch bei schwerer Nierenfunktionseinschränkung oder schwerer pulmonaler Hämorrhagie [35, 37].

Die zugelassene Dosierung für RTX beträgt 4-mal $375 \mathrm{mg} / \mathrm{m}^{2} \mathrm{im}$ wöchentlichen Abstand. Anhand nichtrandomisierter Studien kann eine vergleichbare Effektivität von 2 Gaben a $1000 \mathrm{mg}$ RTX im Abstand von 2 Wochen angenommen werden (rheumatologisches Schema, jedoch „offlabel“).

\section{Remissionsinduktion bei Patienten ohne organbedrohende oder lebensbedrohliche Manifestationen}

Für Patienten ohne organbedrohende oder lebensbedrohliche Manifestation kann eine weniger intensive Immunsuppression erwogen werden. Mycophenolat-Mofetil (MMF; 2-mal 1g) zeigte im Vergleich zu i.v. CYC (Remissionserhaltung in beiden Armen mit Azathioprin [AZA]) eine vergleichbare Remissionsrate, jedoch waren nach 6 Monaten Rezidive in der MMF-Gruppe häufiger und traten v.a. bei PR3-ANCA+Patienten auf [20]. Die Therapie mit MMF stellt einen Off-label-Einsatz dar.

Im lokalisierten bzw. früh-systemischen Stadium mit HNO- bzw. Lungenmanifes- tation empfehlen die Leitlinien auch den Einsatz von MTX (+GC; [11]). Eine eingeschränkte Nierenfunktion stellt eine Kontraindikation dar.

\section{Additiver Einsatz der Plasma- pherese}

Merke. Die Indikation zum Plasmaaustausch muss anhand neuer Studiendaten zurückhaltend gestellt werden. Die Plasmapherese ist bei doppelt positiven Patienten mit Nachweis von ANCA und AntiGBM-Antikörpern Bestandteil der InitialTherapie.

Die Rationale zum Einsatz der Plasmapherese liegt in der Entfernung pathophysiologisch bedeutsamer Autoantikörper. In der MEPEX-Studie, die Patienten mit schwerer Nierenfunktionseinschränkung (Kreatinin $>500 \mu \mathrm{mol} / \mathrm{l}$ ) untersuchte, zeigte der additive Plasmaaustausch (7 Plasmapheresen mit $60 \mathrm{ml} / \mathrm{kg}$ Körpergewicht [KG] in 14 Tagen) zusätzlich zu oralem CYC eine signifikante Reduktion der Dialysepflicht nach 1 Jahr [15]. Jedoch war im Langzeitverlauf (5 Jahre) kein signifikanter Unterschied bezüglich des Erhalts der Nierenfunktion mehr nachweisbar [41]. In der größten Kohortenstudie an 37 Patienten mit diffuser pulmonaler Hämorrhagie konnte ebenfalls kein positiver Effekt der Plasmapherese gezeigt werden [3].

Die PEXIVAS-Studie untersuchte an 704 Patienten mit GPA/MPA und schwerem Organbefall (alveoläre Hämorrhagie und/oder Nierenbeteiligung, glomeruläre Fikltrationsrate [GFR] $<50 \mathrm{ml} / \mathrm{min}$ ) den Effekt der Plasmapherese zusätzlich zur Induktionstherapie mit CYC oder RTX, jeweils in Kombination mit GC [42]. Hinsichtlich des Endpunkts Tod oder terminale Niereninsuffizienz konnte kein signifikanter Nutzen der Plasmapherese belegt werden.

Vor diesem Hintergrund stellen wir die Indikation zur additiven Plasmapherese in der Behandlung der AAV sehr zurückhaltend. Aktuell erscheint der Einsatz der Plasmapherese nur noch bei gleichzeitigem Nachweis von ANCA und Anti-GBM(glomeruläre Basalmembran)Antikörpern notwendig und ist als Ultima Ratio bei fehlendem Therapieansprechen überlegenswert. 


\section{Induktionstherapie bei Rezidiv}

Ein Rezidiv mit schwerer Organbeteiligung sollte wie eine Neuerkrankung behandelt werden [11, 34], RTX wird dabei bevorzugt. Dies gilt besonders für PR3-ANCA+Patienten und/oder eine vorangegangene CYC-Therapie (RAVE, RITAZAREM; [36, 37]).

Die Evidenzlage zur Behandlung von Rezidiven ohne schwere Organbeteiligung ist gering. Eine Erhöhung der GC-Dosis führt in der Regel zum Ansprechen, im Verlauf ist oft eine Intensivierung bzw. Umstellung der immunsuppressiven Therapie zur GC-Einsparung erforderlich. Studiendaten liegen für MMF vor, welches einer Therapie mit oralem CYC (6 Monate, anschließend AZA) nicht unterlegen war [39], allerdings muss konsekutiv mit Rezidiven gerechnet werden.

\section{Therapie bei refraktärer Erkrankung}

Ein Therapieversagen tritt mit den aktuellen Induktionsprotokollen nur noch selten auf $(<10 \%)$. Die Wahl der Zweitlinientherapie richtet sich nach der Vortherapie. Neben einer Steigerung der GC-Dosis wird bei CYC-refraktären Patienten eine Umstellung auf RTX mit guten Erfolgsaussichten empfohlen. Alternativ ist eine Umstellung von der i.v. auf die orale CYC-Gabe möglich. Bei RTX-Versagen kann auf CYC umgestellt werden. Unter einer Kombinationstherapie von CYC und RTX muss mit einer anhaltenden B-Zell-Depletion, Immunglobulinmangel und einem erhöhten Infektionsrisiko gerechnet werden [11]. Der Einsatz der Plasmapherese kann im Einzelfall erwogen werden.

Vor allem bei infektiösen Komplikationen stellt die i.v. Immunglobulingabe eine sinnvolle und verträgliche Therapiealternative dar ( $2 \mathrm{~g} / \mathrm{kg} \mathrm{KG}$ als Einzeldosis oder $0,5 \mathrm{~g} / \mathrm{kg}$ KG für 4 Tage), allerdings ohne länger anhaltenden Therapieeffekt.

\section{Auswahl der Therapie}

In den Leitlinien werden CYC und RTX in Kombination mit GC als Induktionstherapie für GPA und MPA mit organbedrohenden oder lebensbedrohlichen Erkrankungsstadien gleichwertig empfohlen [11]. Klinische, histologische und serologi- sche Risikofaktoren für Komplikationen, eine ungünstige Langzeitprognose und Rezidivneigung sowie die Patientenpräferenz helfen bei der Auswahl.

RTX sollte in folgenden Situationen bevorzugt werden:

- junge Patienten, bei denen der Erhalt der Fertilität von Bedeutung ist;

- Kontraindikationen oder Unverträglichkeit für CYC;

- Angst vor CYC-Nebenwirkungen (z. B. Alopezie);

- Tumoranamnese oder aktive Tumorerkrankung;

- Patienten mit hoher kumulativer CYCDosis;

- unter CYC-Therapie refraktäre Patienten.

RTX zeigt ebenfalls Vorteile bei Patienten mit folgenden Merkmalen:

- PR3-ANCA-Positivität,

- granulomatöse Manifestationen (GPA),

- rezidivierender Verlauf insbesondere nach Vortherapie mit CYC.

CYC und RTX sind gleichwertig bei Patienten mit folgenden Merkmalen:

- MPO-ANCA-Positivität,

- Diagnose einer MPA.

CYC sollte in folgenden Situationen bevorzugt werden:

- ANCA-negative Patienten aufgrund des Fehlens von Studien,

- Hepatitis-B-positive Patienten,

- unter RTX-Therapie refraktäre Patienten.

MMF stellt eine Therapiealternative dar bei folgenden Patienten:

- ohne organ- oder lebensbedrohliche Manifestationen;

- mit organbedrohenden Manifestationen, wenn MPO-ANCA+;

- mit Kontraindikationen für CYC und RTX (und MTX).

\section{Stellenwert der Glukokortikoide}

Merke. Bisher kann auf den Einsatz von Glukokortikoiden in der Induktionstherapie nicht verzichtet werden. Eine rasche Steroidreduktion ist sicher und mit weniger Nebenwirkungen verbunden.
In jedem Krankheitsstadium wird zur Remissionsinduktion eine begleitende GCTherapie empfohlen [11]. Allerdings sind gerade GC für eine Reihe von Nebenwirkungen (erhöhtes Infektionsrisiko, Frühmortalität) verantwortlich.

Einheitlich wird eine initiale GC-Dosis von $1 \mathrm{mg} / \mathrm{kg}$ KG Prednisolonäquivalent/Tag empfohlen (mit einer Begrenzung der Maximaldosis auf 50-75 mg/Tag; [11, 34]). Bei kritischem Organbefall (z.B. rapid-progressive Glomerulonephritis [RPGN], schwere pulmonale Hämorrhagie) können i.v. Methylprednisolonpulse (250-1000 mg für 3 Tage) gegeben werden, um ein rascheres Therapieansprechen zu erreichen. Jedoch ist dies nicht evidenzbasiert und trägt zur Steroidtoxizität bei.

In der PEXIVAS-Studie [42] war eine rasche Steroidreduktion dem bisherigen Standard nicht unterlegen und mit einer geringeren Infektionsrate im ersten Behandlungsjahr verbunden, sodass dieses reduzierte GC-Schema für den klinischen Alltag übernommen werden kann (- Tab. 5). Die Therapie muss an den individuellen Patientenverlauf angepasst werden. Ein schlechteres Therapieansprechen kann eine Dosiserhöhung oder eine langsamere Reduktion erfordern. Ein vollständiges Absetzen der GC innerhalb der ersten 6 Monate ist möglich, führte aber in Studien (RAVE, SCOUT) im Vergleich zu den EUVAS-Daten zu geringeren Remissionsraten und vermehrten Rezidiven [26, 37].

Ein neuer therapeutischer Ansatz zur GC-Einsparung stellt die Therapie mit dem C5a-Rezeptor-Inhibitor Avacopan dar. In einer randomisierten Studie (ADVOCATE) erhielten Patienten mit ANCA+-GPA oder -MPA neben RTX oder CYC (gefolgt von AZA) entweder eine Standard-GC-Therapie (mit Ausschleichen über 20 Wochen) oder Avacopan (2-mal 30 mg, mit Ausschleichen der GC-Therapie über 4 Wochen; [17]). Avacopan zeigte eine vergleichbare Remissionsrate nach 26 Wochen und eine signifikant höhere Rate anhaltender Remissionen nach 52 Wochen, verbunden mit signifikant weniger GC-assoziierten Nebenwirkungen. Avacopan zeigte auch ein verbessertes renales Ansprechen, bezogen auf eGFR und Albuminurie sowie die FatigueSymptomatik $[16,17]$. Aktuell besteht noch 


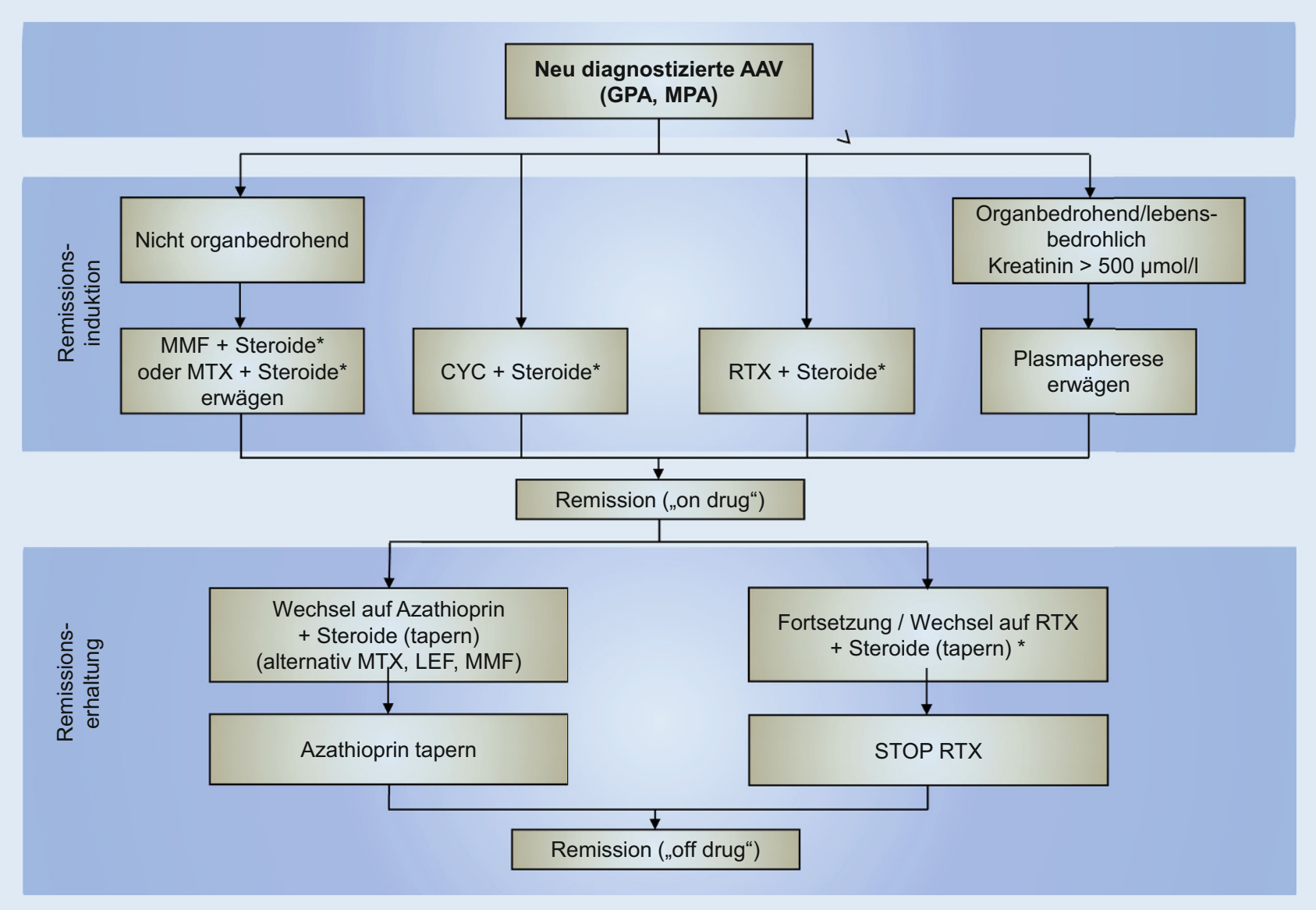

Abb. 1 A Therapeutisches Vorgehen bei ANCA(antineutrophile zytoplasmatische Antikörper)-assoziierter Vaskulitis ( $A A V$; GPA Granulomatose mit Polyangiitis, MPA mikroskopische Polyangiitis mit/ohne extrarenale Manifestationen, MMF Mycophenolat-Mofetil, MTX Methotrexat, CYC Cyclophosphamid, RTX Rituximab, LEF Leflunomid, * Cotrimoxazol als Pneumocystis-Prophylaxe)

keine Zulassung, auch fehlen Daten zur Therapiedauer über 12 Monate hinaus.

Weitere Ansätze zum raschen GC-Ausschleichen, wie eine initiale Kombination von CYC und RTX, wurden in kleineren unkontrollierten Studien untersucht. So konnte in einer Studie bei 49 Patienten mit MPA und GPA durch eine kombinierte CYC-RTX-Therapie (6-mal i.v. CYC-Puls, RTX 2-mal $1 \mathrm{~g}$ ) und eineGC-Therapie über maximal 2 Wochen ein gutes Ansprechen erzielt werden [30]. Allerdings sollte dies durch randomisierte Studien bestätigt werden. Andererseits wird in aktuellen Studien eine verlängerte niedrig dosierte GC-Therapie zur Remissionserhaltung (Prednisolon $5 \mathrm{mg}$ ) untersucht (TAPIR, MAINEPSAN), deren Ergebnisse noch ausstehen.

\section{Remissionserhaltung bei GPA und MPA}

Trotz guter Erfolge der Remissionsinduktion erleidet mindestens die Hälfte der AAVPatienten im Verlauf der Erkrankung ein Rezidiv. Auch nach Induktion mit RTX ist die Rezidivrate nicht niedriger als nach CYC [27]. Das Rezidivrisiko wird von Faktoren wie Krankheitsentität, Klinik, ANCA-Subtyp und gewählter Therapie beeinflusst.

Als Risikofaktoren für ein Rezidiv gelten:

- Diagnose einer GPA, PR3-ANCA;

- Lungen- oder kardiovaskuläre Beteiligung sowie bessere Nierenfunktion;

- frühere Rezidive;

- ein erneut positiver ANCA nach Remissionsinduktion und in geringerem Maße ein persistierender ANCA bzw. ein ANCA-Titer-Anstieg;

- kürzere Therapiedauer;
- geringere CYC-Gesamtdosis;

- frühes Steroidende.

\section{Remissionserhaltung}

Bisher richtete sich die Auswahl der Remissionserhaltung nach der gewählten Induktionstherapie (• Tab. 6; 0 Abb. 1).

Merke. Zur Remissionserhaltung wird eine Therapie mit Rituximab oder Azathioprin, kombiniert mit niedrig dosierten Steroiden, empfohlen.

\section{Remissionserhaltung nach CYC- Induktion}

Die längsten Erfahrungen gibt es mit AZA, das nach einer 3- bis 6-monatigen Induktionstherapie und Erreichen einer Remission die Therapie mit CYC ersetzen kann (CYCAZAREM; [14]). Während im kurzfris- 
Tab. 5 Glukokortikoiddosierung: Standard-und Reduced-dose-Schema, begleitend zu einer Remissionsinduktion während der ersten 6 Behandlungsmonate, entsprechend der Dosierung in der PEXIVAS-Studie. (Nach [42])

\begin{tabular}{|c|c|c|c|c|c|c|}
\hline \multirow[b]{3}{*}{ Woche } & \multicolumn{6}{|c|}{ Prednisolondosis (mg/Tag) } \\
\hline & \multicolumn{3}{|c|}{ Standard } & \multicolumn{3}{|c|}{ „Reduced dose" } \\
\hline & $<50 \mathrm{~kg}$ & $50-75 \mathrm{~kg}$ & $>75 \mathrm{~kg}$ & $<50 \mathrm{~kg}$ & $50-75 \mathrm{~kg}$ & $>75 \mathrm{~kg}$ \\
\hline & Puls & Puls & Puls & Puls & Puls & Puls \\
\hline 1 & 50 & 60 & 75 & 50 & 60 & 75 \\
\hline 2 & 50 & 60 & 75 & 25 & 30 & 40 \\
\hline $3-4$ & 40 & 50 & 60 & 20 & 25 & 30 \\
\hline $5-6$ & 30 & 40 & 50 & 15 & 20 & 25 \\
\hline $7-8$ & 25 & 30 & 40 & 12,5 & 15 & 20 \\
\hline $9-10$ & 20 & 25 & 30 & 10 & 12,5 & 15 \\
\hline $11-12$ & 15 & 20 & 25 & 7,5 & 10 & 12,5 \\
\hline $13-14$ & 12,5 & 15 & 20 & 6 & 7,5 & 10 \\
\hline $15-16$ & 10 & 10 & 15 & 5 & 5 & 7,5 \\
\hline $17-18$ & 10 & 10 & 15 & 5 & 5 & 7,5 \\
\hline $19-20$ & 7,5 & 7,5 & 10 & 5 & 5 & 5 \\
\hline $21-22$ & 7,5 & 7,5 & 7,5 & 5 & 5 & 5 \\
\hline $23-52$ & 5 & 5 & 5 & 5 & 5 & 5 \\
\hline$>52$ & \multicolumn{3}{|c|}{ Nach lokaler Praxis } & \multicolumn{3}{|c|}{ Nach lokaler Praxis } \\
\hline
\end{tabular}

tigen Verlauf die Rezidivrate vergleichbar war, stieg sie im Langzeitverlauf nach Therapieende signifikant an, allerdings ohne Auswirkungen auf Mortalität und Nierenfunktion [10]. Zur Remissionserhaltung war Methotrexat (MTX) vergleichbar effektiv [28], hat sich jedoch im nephrologischen Alltag aufgrund seiner Kontraindikation bei Niereninsuffizienz nicht etabliert. AZA war MMF als Erhaltungstherapie nach CYC-Induktion in Bezug auf Rezidive überlegen [13], somit kann MMF nur als Reservemedikation empfohlen werden. Bei eingeschränkter Studienlage stellt auch Leflunomid ein Reserveimmunsuppressivum dar. Nebenwirkungen (z.B. Polyneuropathie, schwerer Hypertonus) schränken den Einsatz ein.

Nach CYC-Induktion war RTX in einer prospektiven, randomisierten Studie (MAINRITSAN; [9]) nach 28 Monaten einer Remissionserhaltung mit AZA klar überlegen. In der RTX-Gruppe traten bei gleicher Häufigkeit von Nebenwirkungen signifikant weniger Rezidive auf. Eine individualisierte Steuerung der RTX-Gaben in Abhängigkeit vom Wiederanstieg der B-Zellen und/oder ANCA ist möglich (MAINRITSAN 2), aber mit einem höheren Aufwand für Kontrolluntersuchungen verbunden und hat sich in der Praxis nicht durchgesetzt [4]. RTX ist in Deutschland seit Ende 2018 auch zur Erhaltungstherapie bei MPA und GPA zugelassen.

\section{Remissionserhaltung nach RTX- Induktion}

In der RITAZAREM-Studie wurde erstmals nach RTX-Induktion der Einsatz von RTX oder AZA zur Remissionserhaltung untersucht, für beide gab es bis dato keine Studien. Eingeschlossen waren Patienten mit Rezidiv einer GPA oder MPA, und es wurde ein intensiviertes RTX-Regime benutzt (RTX $1 \mathrm{~g}$ alle 4 Monate). Während die Daten zur Induktionsphase bereits veröffentlich wurden [36], stehen die Ergebnisse der Erhaltungsphase noch aus.

\section{Dauer der Remissionserhaltung}

Merke. Eine längere Erhaltungstherapie bedeutet weniger Rezidive, jedoch auch mehr Nebenwirkungen.

Nach Absetzen der Erhaltungstherapie zeigt sich sowohl für AZA- als auch für RTXbehandelte Patienten ein Anstieg der Zahl der Rezidive [38]. Dies begründet Studien mit verlängerter Dauer der Remissionserhaltung. Eine 4-jährige Therapie mit AZA war für den Erhalt der Remission und der Nierenfunktion signifikant effektiver als eine Therapie über 22 Monate [21]. Ähnli- ches zeigt sich auch für eine Verlängerung der Therapiedauer von RTX über 46 Monate [5]. Andererseits tritt bei etwa $50 \%$ der Patienten kein Rezidiv auf. Auch kann sich ein Rezidiv erst Jahre nach Beendigung der Therapie zeigen. Zudem sind die Nebenwirkungen unter einer Langzeitimmunsuppression mit AZA (z. B. Tumorrisiko, Knochenmarktoxizität), aber auch mit RTX (Hypogammaglobulinämie, Leukopenien) sowie die Kosten zu berücksichtigen.

Die optimale Dauer der Erhaltungstherapie ist unklar. Das Rezidivrisiko sollte bei der Auswahl sowohl des Medikaments als auch der Therapiedauer berücksichtigt werden, dies ist in bisherigen Studien nicht erfolgt. Bei Patienten mit Risikofaktoren für ein Rezidiv wird bevorzugt RTX zur Remissionserhaltung eingesetzt, insbesondere bei Patienten mit Rezidiv, PR3-ANCA, AZA-Unverträglichkeit und nach einer RTX-Induktion. Auch muss eine Verlängerung der Therapiedauer auf 48 Monate oder länger diskutiert werden. Bisher fehlen systematische Untersuchungen zu optimaler Dosis $(0,5-1 \mathrm{~g})$, Infusionsabständen (4 bis 6 Monate) sowie Dauer der RTX-Therapie.

\section{Therapie der EGPA}

Die Studienlage ist eingeschränkt. In frühen Studien wurden zudem Patienten mit EGPA und MPA/Polyarteritis nodosa (PAN) gemeinsam untersucht. Hinzu kommt eine klinische Heterogenität mit vaskulitisassoziierten (meist MPO-ANCA+) gegenüber eosinophilenvermittelten Organbeteiligungen. Für die Auswahl der Therapie wird eine Einteilung der Schwere der Erkrankung gemäß FFS vorgenommen. Basis der Therapie ist der Einsatz von GC (0,5-1,0 mg/kg KG Prednisolon; [22]).

Für Patienten mit schwerer Organmanifestation (FFS $\geq 2$ ), insbesondere bei neurologischer und kardialer Manifestation, wird die zusätzliche Gabe von CYC empfohlen (Puls oder oral), dies allerdings auf Basis von Kohortenstudien und Metaanalysen [46]. Die optimale Therapiedauer ist unklar, eine längere Therapiedauer ( 12 vs. 6 Monate) war mit weniger Rezidiven verbunden. Im Anschluss wird eine Erhaltungstherapie mit AZA in Kombination mit GC empfohlen, MTX und Leflunomid sind als Alternativen möglich. Patienten mit weniger schweren Krankheitsverläufen (FFS <2) werden pri- 


\begin{tabular}{|c|c|c|c|c|c|}
\hline $\begin{array}{l}\text { Therapie (Stu- } \\
\text { dienprotokoll) }\end{array}$ & Protokoll & Dosis & Ergebnis & Zu beachten & Zulassung \\
\hline $\begin{array}{l}\text { AZA } \\
\text { (CYCAZAREM) } \\
{[14]}\end{array}$ & $\begin{array}{l}\text { Nach Induktion mit } \\
\text { CYC, AZA vs. CYC bis } \\
\text { Monat } 12\end{array}$ & $\begin{array}{l}2 \mathrm{mg} / \mathrm{kg} \text { p.o., Beginn } 14 \text { Tage } \\
\text { nach letztem CYC-Puls, im } \\
\text { Verlauf Taper, nach } 12 \mathrm{Mo}- \\
\text { naten auf } 1,5 \mathrm{mg} / \mathrm{kg}, \mathrm{nach} \\
18 \text { Monaten auf } 1 \mathrm{mg} / \mathrm{kg}\end{array}$ & $\begin{array}{l}\text { Weniger NW als CYC, } \\
\text { aber mehr Rezidive }\end{array}$ & $\begin{array}{l}\text { Keine Kombination mit Al- } \\
\text { lopurinol oder Febuxostat, } \\
\text { NW beachten, Laborkon- } \\
\text { trollen obligat }\end{array}$ & $\begin{array}{l}\text { Formal nicht } \\
\text { zugelassen }\end{array}$ \\
\hline $\begin{array}{l}\text { AZA (REMAIN) } \\
{[21]}\end{array}$ & $\begin{array}{l}\text { Nach Induktion mit CYC, } \\
\text { AZA } 48 \text { vs. } 24 \text { Monate }\end{array}$ & - & $\begin{array}{l}\text { Weniger Rezidive mit } \\
\text { längerer Therapie }\end{array}$ & - & - \\
\hline $\begin{array}{l}\text { MTX (WEGENT) } \\
{[28,31]}\end{array}$ & $\begin{array}{l}\text { Nach Induktion mit } \\
\text { CYC, AZA vs. MTX für } \\
12 \text { Monate }\end{array}$ & $\begin{array}{l}0,3 \mathrm{mg} / \mathrm{kg} \mathrm{KG}, 1-\mathrm{mal} \text { pro } \\
\text { Woche }(15-25 \mathrm{mg})\end{array}$ & $\begin{array}{l}\text { Vergleichbare Rate } \\
\text { an NW und Rezidiven }\end{array}$ & $\begin{array}{l}\text { Folsäure am Tag nach MTX- } \\
\text { Gabe, kontraindiziert bei } \\
\text { Niereninsuffizienz }\end{array}$ & $\begin{array}{l}\text { Formal nicht } \\
\text { zugelassen }\end{array}$ \\
\hline $\begin{array}{l}\text { MMF (IMPROVE) } \\
\text { [13] }\end{array}$ & $\begin{array}{l}\text { Nach Induktion mit } \\
\text { CYC, AZA vs. MMF für } \\
12 \text { Monate }\end{array}$ & $\begin{array}{l}\text { 2-mal } 1 \text { g p.o., im Verlauf } \\
\text { Taper }\end{array}$ & $\begin{array}{l}\text { Höhere Rezidivrate } \\
\text { unter MMF, ver- } \\
\text { gleichbare NW }\end{array}$ & - & "Off-label" \\
\hline $\begin{array}{l}\text { RTX } \\
\text { (MAINRITSAN) } \\
{[9,38]}\end{array}$ & $\begin{array}{l}\text { Nach Induktion mit } \\
\text { CYC, RTX vs. AZA für } \\
24 \text { Monate }\end{array}$ & $\begin{array}{l}\text { 2-mal } 500 \text { mg i.v. zu Beginn, } \\
\text { dann } 500 \text { mg RTX i.v. alle } \\
6 \text { Monate }\end{array}$ & $\begin{array}{l}\text { Weniger Rezidive } \\
\text { unter RTX, NW ver- } \\
\text { gleichbar }\end{array}$ & $\begin{array}{l}\text { Risiko für Hypogammaglo- } \\
\text { bulinämie }\end{array}$ & $\begin{array}{l}\text { Zugelassen } \\
\text { für GPA und } \\
\text { MPA, für } 2 \text { bis } \\
5 \text { Jahre }\end{array}$ \\
\hline $\begin{array}{l}\text { RTX } \\
\text { (MAINRITSAN2) } \\
{[4]}\end{array}$ & $\begin{array}{l}\text { Nach Induktion mit } \\
\text { CYC, RTX fixe Gabe vs. } \\
\text { Tailored-Gabe nach } \\
\text { B-Zellen/ANCA }\end{array}$ & - & $\begin{array}{l}\text { Vergleichbare Re- } \\
\text { zidivrate, weniger } \\
\text { Infusionen }\end{array}$ & $\begin{array}{l}\text { Engmaschige Kontrollen } \\
\text { notwendig }\end{array}$ & - \\
\hline $\begin{array}{l}\text { RTX } \\
\text { (MAINRITSAN3) } \\
{[5]}\end{array}$ & $\begin{array}{l}\text { Nach Induktion mit CYC, } \\
\text { RTX } 24 \text { vs. } 48 \text { Monate }\end{array}$ & $500 \mathrm{mg}$ RTX i.v. alle 6 Monate & $\begin{array}{l}\text { Weniger Rezidive bei } \\
\text { längerer Therapie- } \\
\text { dauer }\end{array}$ & - & - \\
\hline $\begin{array}{l}\text { RTX } \\
\text { (RITAZAREM) } \\
{[36]}\end{array}$ & $\begin{array}{l}\text { Patienten mit Rezidiv } \\
\text { GPA/MPA, nach Indukti- } \\
\text { on mit RTX, RTX vs. AZA } \\
\text { für } 24 \text { Monate }\end{array}$ & $\begin{array}{l}1000 \mathrm{mg} \text { RTX i.v. alle } 4 \text { Mona- } \\
\text { te }\end{array}$ & $\begin{array}{l}\text { Weniger Rezidive } \\
\text { unter RTX, NW ver- } \\
\text { gleichbar }\end{array}$ & - & $\begin{array}{l}\text { Dosis von } \\
1000 \text { mg for- } \\
\text { mal "off-label" }\end{array}$ \\
\hline $\begin{array}{l}\text { Avacopan } \\
\text { (ADVOCATE) } \\
{[17]}\end{array}$ & $\begin{array}{l}\text { ANCA+-GPA und -MPA } \\
\text { unter Therapie mit CYC } \\
\text { (gefolgt von AZA) oder } \\
\text { RTX }\end{array}$ & 2-mal $30 \mathrm{mg}$ p.o./Tag & $\begin{array}{l}\text { Avacopan mit bes- } \\
\text { serer Remissionsrate } \\
\text { nach } 12 \text { Monaten, } \\
\text { weniger GC-NW }\end{array}$ & $\begin{array}{l}\text { Bisher keine Langzeiterfah- } \\
\text { rung }>12 \text { Monate }\end{array}$ & $\begin{array}{l}\text { Bisher nicht } \\
\text { zugelassen } \\
\text { bzw. nicht } \\
\text { verfügbar }\end{array}$ \\
\hline
\end{tabular}

mär mit GC behandelt. Die Datenlage für die Gabe von AZA oder MTX ist nicht überzeugend, auch wenn ihr Einsatz im Alltag oft erfolgt [46].

Der Gabe von RTX hat in nichtrandomisierten Studien ein verbessertes Ansprechen gezeigt und kann v. a. für ANCA+Patienten mit vaskulitischen Organmanifestationen erwogen werden [22]. Topische GC kommen für die Kontrolle der Krankheitsaktivität im oberen und unteren Respirationstrakt zum Einsatz.

Im Langzeitverlauf sind v.a. Rezidive, eine unzureichende Kontrolle der Asthmakomponente und Steroidnebenwirkungen häufig. Die beim eosinophilen Asthma wirksame Therapie mit Anti-IL-5-Antikörpern wurde auch bei der EGPA getestet. Mepolizumab (300 mg s.c. alle 4 Wochen) erzielte in einer randomisierten Studie (MIRRA) bei Patienten mit Rezidiv bzw.
Therapierefraktärität eine bessere Krankheitskontrolle und ermöglichte eine Steroidreduktion [44]. Allerdings konnte bei etwa der Hälfte der Patienten keine komplette Remission erreicht werden, und nach Absetzen der Therapie traten gehäuft Rezidive auf. Die für das therapierefraktäre eosinophile Asthma zugelassene Dosis (100 mg s.c. alle 4 Wochen) war in Fallserien ebenfalls effektiv. Eine Zulassung der European Medicines Agency (EMA) für die Behandlung der EGPA fehlt bisher. Auch liegen keine Studien zum Einsatz bei Erstmanifestation bzw. schwerer Organmanifestation der EGPA vor. In Fallserien wurden auch der Anti-IL-5-Rezeptor-Antikörper Benralizumab und der Anti-IL-5-Antikörper Reslizumab erfolgreich bei EGPA eingesetzt, die beide aktuell in randomisierten Studien untersucht werden.

\section{Zukünftige Therapiestrategien}

Auch wenn es gelungen ist, das Ansprechen zu optimieren und Nebenwirkungen zu reduzieren, sind weiterhin neue Therapien und Behandlungsstrategien gefragt. So haben bisherige Therapiestudien Patienten mit GPA und MPA bzw. PR3- und MPO-ANCA in der Regel gemeinsam untersucht und Unterschiede im Ansprechen und bezüglich des Rezidivrisikos ignoriert.

Aktuelle und zukünftige Studien zur Therapieoptimierung sind auf eine verbesserte B-Zell-Elimination (Obinutuzumab), die Kombination von B-Zell-Depletion und BAFF-Inhibition (Rituximab + Belimumab zur Remissionsinduktion, COMBIVAS), Plasmazellen als Therapietarget (AntiCD38-Therapie, Daratumumab) oder eine Kostimulationsblockade (Abatacept, ABROGATE) ausgerichtet. Als weitere The- 
Tab. 7 Supportive Maßnahmen

\begin{tabular}{|c|c|}
\hline Risikofaktor & Empfohlene Maßnahmen \\
\hline \multirow[t]{3}{*}{ Rituximab (RTX) } & $\begin{array}{l}\text { Infektionsscreening vor Therapie (aktive Hepatitis B als Kontraindikation, } \\
\text { ggf. Prophylaxe bei abgelaufener Hepatitis B, cave bei HIV-Infektion) }\end{array}$ \\
\hline & $\begin{array}{l}\text { Überprüfung Impfstatus (nach B-Zell-Depletion eingeschränkte Impfant- } \\
\text { wort) }\end{array}$ \\
\hline & Risiko Hypogammaglobulinämie \\
\hline \multirow[t]{6}{*}{$\begin{array}{l}\text { Cyclophos- } \\
\text { phamid (CYC) }\end{array}$} & $\begin{array}{l}\text { Patienten im gebär-/zeugungsfähigen Alter: Beratung vor Therapie, Kryo- } \\
\text { konservierung von Eizellen/Sperma anbieten, Therapie mit GnRH-Analoga } \\
\text { für Frauen im gebärfähigen Alter }\end{array}$ \\
\hline & $\begin{array}{l}\text { Ausreichende Flüssigkeitsgabe am Tag der CYC-Gabe (cave: nur bei ausrei- } \\
\text { chender Diurese) }\end{array}$ \\
\hline & Mesna am Tag der CYC-Gabe \\
\hline & Bestimmung Leukozytennadir und Dosisanpassung \\
\hline & Malignomscreening entsprechend nationalen Leitlinien \\
\hline & Früherkennung hämorrhagische Zystitis/Urothelkarzinom \\
\hline \multirow[t]{4}{*}{ Infektionen } & $\begin{array}{l}\text { Pneumocystis-Prophylaxe mit Trimethoprim-Sulfamethoxazol ( } 800 / 160 \mathrm{mg} \\
\text { 3-mal pro Woche oder } 400 / 80 \mathrm{mg} \text { täglich), insbesondere unter CYC- und } \\
\text { RTX-Therapie mit GC-Dosen > } 10 \text { (15) } \mathrm{mg} / \mathrm{Tag}\end{array}$ \\
\hline & $\begin{array}{l}\text { Überprüfung Impfstatus, Schutzimpfungen entsprechend nationalen Emp- } \\
\text { fehlungen, Impfung gegen Pneumokokken und Influenza }\end{array}$ \\
\hline & Screening für Hepatitis und HIV \\
\hline & Tuberkulosescreening erwägen \\
\hline \multirow[t]{3}{*}{ Kardiovaskulär } & $\begin{array}{l}\text { Evaluation des kardiovaskulären Risikos, präventive Maßnahmen entspre- } \\
\text { chend nationalen Leitlinien }\end{array}$ \\
\hline & Thrombembolieprophylaxe bei hoher entzündlicher Aktivität \\
\hline & Antiproteinurische Therapie mit ACE-Hemmer/ARB \\
\hline Osteoporose & Entsprechend nationalen Leitlinien \\
\hline
\end{tabular}

rapie des Komplementsystems wird der monoklonale Antikörper gegen C5a (Vilobelimab [IFX-1]) getestet. Ein interessanter Ansatz ist die pharmakologische Inhibition von Cathepsin C, die zu einer verminderten Expression von PR3 in neutrophilen Granulozyten führt.

\section{Supportive Maßnahmen}

In den letzten Jahren ist die Bedeutung präventiver Maßnahmen in den Fokus gerückt. Insbesondere Infektionen, kardiovaskuläre Ereignisse und Malignome sind kurz- bzw. langfristig von Bedeutung (• Tab. 7). Hervorzuheben sind:

- Hepatitis-B-Screening vor RTX-Gabe;

- Uroprotektion und Erhalt der Fertilität unter CYC;

- Einsatz von Trimethoprim-Sulfamethoxazol zur Prophylaxe einer Pneumocystis-jiroveci-Pneumonie (PJP) für die Dauer der Remissionsinduktion mit CYC, RTX und/oder eine höher dosierte GC-Therapie (> 10-15 mg Prednisolon-

Möglicherweise ist auch eine längere Gabe von Trimethoprim-Sulfamethoxazol sinnvoll. In einer Kohorte von mit RTX behandelten Patienten zeigte sich eine Halbierung des Risikos schwerer Infektionen [23].

Alle Impfungen sollten entsprechend den STIKO(Ständige Impfkommission am Robert-Koch-Institut)-Empfehlungen erfolgen (insbesondere gegen Pneumokokken und Influenza). Die Rezidivrate wird durch die Impfungen nicht beeinflusst. Die Wirksamkeit einer Impfung kann in Abhängigkeit von der Intensität der immunsuppressiven Therapie (insbesondere unter RTX) stark eingeschränkt sein. Dies ist gerade in der aktuellen COVID-19(, ronavirus disease 2019")-Pandemie von besonderer Bedeutung.

Ob der Einsatz von SGLT2 (",sodium-glucose linked transporter 2")-Hemmern bei AAV-Patienten die kardiovaskuläre Morbi- dität und Mortalität sowie die Progression der Nierenerkrankung positiv beeinflussen kann, muss in zukünftigen Studien beurteilt werden.

\section{Fazit für Praxis}

- ANCA(antineutrophile zytoplasmatische Antikörper)-assoziierte Vaskulitiden (AAV) sind entzündliche Systemerkrankungen mit häufiger Manifestation im Bereich von Respirationstrakt und Nieren.

- Die Therapie von AAV ist biphasisch und wird nach Krankheitsschwere und Rezidivrisiko ausgewählt.

- Zur Remissionsinduktion bei Granulomatose mit Polyangiitis (GPA)/mikroskopischer Polyangiitis (MPA) mit organbedrohender Erkrankung werden Cyclophosphamid (CYC) und Rituximab (RTX) in Kombination mit Glukokortikoiden (GC) eingesetzt, die B-Zell-depletierende Therapie mit RTX gewinnt weiter an Bedeutung.

- Für GPA/MPA-Patienten ohne organbedrohende Manifestation sind Methotrexat und Mycophenolat-Mofetil in Kombination mit GC wirksam.

- Eine schnellere Reduktion der GC-Exposition ist laut aktuellen Studiendaten möglich. Neue Therapieansätze (z. B. Avacopan) könnten in Zukunft eine weitere GCEinsparung, ggf. sogar eine langfristige Steroidfreiheit ermöglichen.

- Der Einsatz der Plasmapherese muss anhand neuer Studiendaten zurückhaltend gesehen werden.

- Zur Remissionserhaltung bei GPA/MPA stehen RTX und Azathioprin zur Verfügung. Auswahl und Therapiedauer richten sich insbesondere nach dem Rezidivrisiko.

- Die Therapie der eosinophilen Granulomatose mit Polyangiitis ist weniger evidenzbasiert und beinhaltet GC, Immunsuppressiva je nach Erkrankungsschwere und zunehmend Biologika (Interleukin[IL]-5-Hemmung).

- Supportive Maßnahmen (u.a. Pneumocystis-Prophylaxe und Impfungen) können Nebenwirkungen reduzieren. 
Korrespondenzadresse

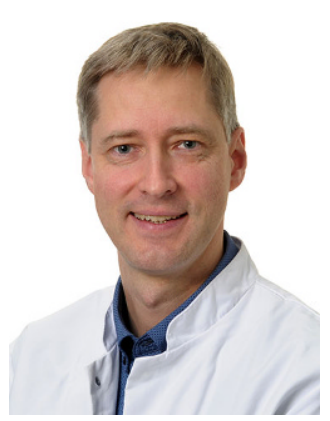

Dr. Ulf Schönermarck

Medizinische Klinik und Poliklinik IV, Nephrologisches Zentrum, Campus Großhadern, LMU Klinikum

Marchioninistr. 15, 81377 München, Deutschland

ulf.schoenermarck@med.uni-muenchen.de

\section{Einhaltung ethischer Richtlinien}

Interessenkonflikt. U. Schönermarck hat Beratungsund Referentenhonorare sowie Forschungsunterstützung von ChemoCentryx/Vifor erhalten. V. Vielhauer hat Beratungs- und Referentenhonorare, Unterstützung für Kongressreisen und Forschungsunterstützung von ChemoCentryx/Vifor erhalten.

Für diesen Beitrag wurden von den Autoren keine Studien an Menschen oder Tieren durchgeführt. Für die aufgeführten Studien gelten die jeweils dort angegebenen ethischen Richtlinien.

\section{Literatur}

1. Berden $A E$, Ferrario $F$, Hagen EC et al (2010) Histopathologic classification of ANCA-associated glomerulonephritis. J Am Soc Nephrol 21:1628-1636

2. Bossuyt X, Cohen Tervaert JW, Arimura $Y$ et al (2017) Position paper: revised 2017 international consensus on testing of ANCAs in granulomatosis with polyangiitis and microscopic polyangiitis. Nat Rev Rheumatol 13:683-692

3. Cartin-Ceba R, Diaz-Caballero L, Al-Qadi MO et al (2016) Diffuse alveolar hemorrhage secondary to antineutrophil cytoplasmic antibodyassociated vasculitis: predictors of respiratory failure and clinical outcomes. Arthritis Rheumatol 68:1467-1476

4. Charles P, Terrier B, Perrodeau É et al (2018) Comparison of individually tailored versus fixedschedule rituximab regimen to maintain ANCAassociated vasculitis remission: results of a multicentre, randomised controlled, phase III trial (MAINRITSAN2). Ann Rheum Dis 77:1143-1149

5. Charles P, Perrodeau E, Samson M et al (2020) Long-term Rituximab use to maintain remission of antineutrophil cytoplasmic antibody-associated vasculitis: a randomized trial. Ann Intern Med 173:179-187

6. de Groot K, Rasmussen N, Bacon PA et al (2005) Randomized trial of cyclophosphamide versus methotrexate for induction of remission in early systemic antineutrophil cytoplasmic antibody-associated vasculitis. Arthritis Rheum 52:2461-2469

7. de Groot K, Harper L, Jayne DR et al (2009) Pulse versus daily oral cyclophosphamide for induction of remission in antineutrophil cytoplasmic antibody-associated vasculitis: a randomized trial. Ann Intern Med 150:670-680

8. Flossmann O, Berden A, De Groot K et al (2011) Long-term patient survival in ANCA-associated vasculitis. Ann Rheum Dis 70:488-494

9. Guillevin L, Pagnoux C, Karras A et al (2014) Rituximab versus azathioprine for maintenance in ANCA-associated vasculitis. $\mathrm{N}$ Engl J Med 371:1771-1780

10. Harper L, Morgan MD, Walsh M et al (2012) Pulse versus daily oral cyclophosphamide for induction of remission in ANCA-associated vasculitis: longterm follow-up. Ann Rheum Dis 71:955-960

11. Hellmich B (2019) ANCA-associated vasculitides: state of the art. ZRheumatol 78:518-528

12. Herlyn K, Buckert F, Gross WL et al (2014) Doubled prevalence rates of ANCA-associated vasculitides and giant cell arteritis between 1994 and 2006 in northern Germany. Rheumatology 53:882-889

13. Hiemstra TF, Walsh M, Mahr A et al (2010) Mycophenolate mofetil vs azathioprine for remission maintenance in antineutrophil cytoplasmic antibody-associated vasculitis: a randomized controlled trial. JAMA 304:2381-2388

14. Jayne D, Rasmussen N, Andrassy K et al (2003) A randomized trial of maintenance therapy for vasculitis associated with antineutrophil cytoplasmic autoantibodies. N Engl J Med 349:36-44

15. Jayne DR, Gaskin G, Rasmussen N et al (2007) Randomized trial of plasma exchange or highdosage methylprednisolone as adjunctive therapy for severe renal vasculitis. J Am Soc Nephrol 18:2180-2188

16. Jayne DRW, Bruchfeld AN, Harper L et al (2017) Randomized trial of $\mathrm{C} 5 \mathrm{a}$ receptor inhibitor avacopan in ANCA-associated vasculitis. J Am Soc Nephrol 28:2756-2767

17. Jayne DRW, Merkel PA, Schall TJ et al (2021) Avacopan for the treatment of ANCA-associated vasculitis. NEngl J Med 384:599-609

18. Jennette JC, Falk RJ, Bacon PA et al (2013) 2012 revised international chapel hill consensus conference nomenclature of vasculitides. Arthritis Rheum 65:1-11

19. Jones RB, Tervaert JW, Hauser T et al (2010) Rituximab versus cyclophosphamide in ANCA-associated renal vasculitis. NEngl J Med 363:211-220

20. Jones RB, Hiemstra TF, Ballarin J et al (2019) Mycophenolate mofetil versus cyclophosphamide for remission induction in ANCA-associated vasculitis: a randomised, non-inferiority trial. Ann Rheum Dis 78:399-405

21. Karras A, Pagnoux C, Haubitz M et al (2017) Randomised controlled trial of prolonged treatment in the remission phase of ANCA-associated vasculitis. Ann Rheum Dis 76:1662-1668

22. Kitching AR, Anders HJ, Basu N et al (2020) ANCAassociated vasculitis. Nat Rev Dis Primers 6:71

23. Kronbichler A, Kerschbaum J, Gopaluni $S$ et al (2018) Trimethoprim-sulfamethoxazole prophylaxis prevents severe/life-threatening infections following rituximab in antineutrophil cytoplasm antibody-associated vasculitis. Ann Rheum Dis 77:1440-1447

24. Little MA, Nightingale $P$, Verburgh $C A$ et al (2010) Early mortality in systemic vasculitis: relative contribution of adverse events and active vasculitis. Ann Rheum Dis 69:1036-1043

25. Miloslavsky EM, Naden RP, Bijlsma JW et al (2017) Development of a Glucocorticoid Toxicity Index (GTI) using multicriteria decision analysis. Ann Rheum Dis 76:543-546

26. Miloslavsky EM, Niles JL, Wallace ZS et al (2018) Reducing glucocorticoid duration in ANCAassociated vasculitis: a pilot trial. Semin Arthritis Rheum 48:288-292

27. Mukhtyar C, Guillevin L, Cid MC et al (2009) EULAR recommendations for the management of primary small and medium vessel vasculitis. Ann Rheum Dis 68:310-317

28. Pagnoux C, Mahr A, Hamidou MA et al (2008) Azathioprine or methotrexate maintenance for ANCA-associated vasculitis. N Engl J Med 359:2790-2803

29. Pagnoux C, Quéméneur T, Ninet J et al (2015) Treatment of systemic necrotizing vasculitides in patients aged sixty-five years or older: results of a multicenter, open-label, randomized controlled trial of corticosteroid and cyclophosphamidebased induction therapy. Arthritis Rheumatol 67:1117-1127

30. Pepper RJ, McAdoo SP, Moran SM et al (2019) A novel glucocorticoid-free maintenance regimen for anti-neutrophil cytoplasmantibody-associated vasculitis. Rheumatology 58:260-268

31. Puéchal X, Pagnoux C, Perrodeau É et al (2016) Long-term outcomes among participants in the WEGENT trial of remission-maintenance therapy for granulomatosis with polyangiitis (Wegener's) or microscopic Polyangiitis. Arthritis Rheumatol 68:690-701

32. Rhee RL, Hogan SL, Poulton CJ et al (2016) Trends in long-term outcomes among patients with antineutrophil cytoplasmic antibody-associated vasculitis with renal disease. Arthritis Rheumatol 68:1711-1720

33. Robson J, Doll H, Suppiah R et al (2015) Damage in the anca-associated vasculitides: long-term data from the European vasculitis study group (EUVAS) therapeutic trials. Ann Rheum Dis 74:177-184

34. Schönermarck U, Gross WL, de Groot K (2014) Treatment of ANCA-associated vasculitis. Nat Rev Nephrol 10:25-36

35. Shah S, Hruskova Z, Segelmark M et al (2015) Treatment of severe renal disease in ANCA positive and negative small vessel vasculitis with rituximab. Am J Nephrol 41:296-301

36. Smith RM, Jones RB, Specks U et al (2020) Rituximab as therapy to induce remission after relapse in ANCA-associated vasculitis. Ann Rheum Dis 79:1243-1249

37. Stone JH, Merkel PA, Spiera Ret al (2010) Rituximab versus cyclophosphamide for ANCA-associated vasculitis. NEngl J Med 363:221-232

38. Terrier B, Pagnoux C, Perrodeau É et al (2018) Longterm efficacy of remission-maintenance regimens for ANCA-associated vasculitides. Ann Rheum Dis 77:1150-1156

39. Tuin J, Stassen PM, Bogdan DI et al (2019) Mycophenolate mofetil versus cyclophosphamide for the induction of remission in nonlife-threatening relapses of antineutrophil cytoplasmic antibodyassociated Vasculitis: randomized, controlled trial. Clin J Am Soc Nephrol 14:1021-1028

40. Unizony S, Villarreal M, Miloslavsky EM et al (2016) Clinical outcomes of treatment of anti-neutrophil cytoplasmic antibody (ANCA)associated vasculitis based on ANCA type. Ann Rheum Dis 75:1166-1169 
41. Walsh M, Casian A, Flossmann O et al (2013) Longterm follow-up of patients with severe ANCAassociated vasculitis comparing plasma exchange to intravenous methylprednisolone treatment is unclear. Kidney Int 84:397-402

42. Walsh M, Merkel PA, Peh CA et al (2020) Plasma exchange and glucocorticoids in severe ANCAassociated vasculitis. N Engl J Med 382:622-631

43. Watts RA, ScottDG, Jayne DR et al (2008) Renal vasculitis in Japan and the UK-are there differences in epidemiology and clinical phenotype? Nephrol Dial Transplant 23:3928-3931

44. Wechsler ME, Akuthota P, Jayne D et al (2017) Mepolizumab or placebo for eosinophilic granulomatosis with polyangiitis. N Engl J Med 376:1921-1932

45. Xiao H, Schreiber A, Heeringa P et al (2007) Alternative complement pathway in the pathogenesis of disease mediated by anti-neutrophil cytoplasmic autoantibodies. Am J Pathol 170:52-64

46. Yates M, Watts RA, Bajema IM et al (2016) EULAR/ERA-EDTA recommendations for the management of ANCA-associated vasculitis. Ann Rheum Dis 75:1583-1594

\section{Strategies for effective treatment of ANCA-associated vasculitides with fewer side effects}

The pathogenesis of anti-neutrophil cytoplasmic antibody (ANCA)-associated vasculitides is complex; however, a better understanding in recent years has enabled new therapeutic approaches. In recent years priority was given to the minimization of treatment-associated toxicity. For induction of remission of severe granulomatosis with polyangiitis (GPA) and microscopic polyangiitis (MPA), glucocorticoids are used as well as cyclophosphamide and rituximab. The current recommendations enable a more rapid tapering of steroid dose and advise caution in the use of plasmapheresis. Rituximab and azathioprine are available options for maintenance of remission. The choice of medication and duration of remission maintenance are oriented particularly to the risk of recurrence. The importance of low-dose steroids has not yet been finally clarified. New treatment approaches, such as the C5a receptor inhibitor avacopan could enable a minimized steroid treatment in the future. The treatment of eosinophilic granulomatosis with polyangiitis (EGPA) is less evidence-based and consists of glucocorticoids, immunosuppressive agents depending on the severity and increasingly more biologics, e.g. interleukin-5 blockade. Supportive measures (e.g. vaccinations, infection prophylaxis, cardiovascular risk management) are increasing in importance. Future treatment strategies must take the individual risk (e.g. ANCA subtype, relapse risk) more into consideration for selection and duration of treatment.

\section{Keywords}

Anti-neutrophil cytoplasmic antibodies - Glucocorticoids . Granulomatosis with polyangiitis . Microscopic polyangiitis · Eosinophilic granulomatosis with polyangiitis

\section{Corona-Webinar: Von Impf-Booster bis Therapie}

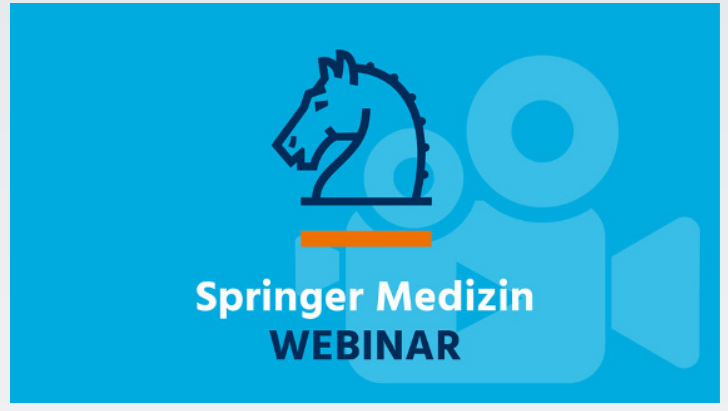

Boostern, Impfquoten, Durchbruchinfektionen: Auch in der vierten Welle der Coronapandemie steht die Vakzine im Fokus, denn „Bei der Therapie sind wir noch längst nicht so weit, wie wir sein wollen." Prof. Bernd Salzberger blickt im Webinar auf die für den kommenden Coronawinter relevanten Studiendaten - und die Lage in den Kliniken: Wer kommt ins Krankenhaus, wer liegt auf Intensiv und wer verstirbt? Die Empfehlungen des Infektiologen für das Vorgehen in den nächsten Wochen sind klar.
Der Referent: Prof. Dr. Bernd Salzberger

- Bereichsleiter Infektiologie, Abteilung für Krankenhaushygiene und Infektiologie am Universitätsklinikum Regensburg - Präsident der Deutschen Gesellschaft für Infektiologie (DGI). - Langjähriger Herausgeber der Zeitschrift Der Internist mit besonderem Fokus auf die Betreuung infektiologischer Themen.

Das Video und alle Empfehlungen finden Sie nach Registrierung kostenfrei hier:

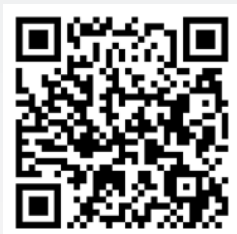

https://www.springermedizin.de/link/19836182 